\title{
3rd International Meeting on Cryptic Chromosomal Rearrangements in Mental Retardation and Autism
}

\author{
Corrado Romano*,1, Samantha JL Knight ${ }^{2}$, Christa Lese Martin ${ }^{3}$ and Bert BA de Vries ${ }^{4}$ \\ ${ }^{1}$ Unit of Pediatrics and Medical Genetics, Department for Mental Retardation, IRCCS Associazione Oasi Maria \\ Santissima, Troina, Italy; ${ }^{2}$ Wellcome Trust Centre for Human Genetics, Oxford, UK; ${ }^{3}$ Department of Human Genetics, \\ Emory University School of Medicine, Atlanta, GA, USA; ${ }^{4}$ Department of Human Genetics, Radboud University Medical \\ Centre, Nijmegen, The Netherlands
}

European Journal of Human Genetics (2007) 15, 1098-1101; doi:10.1038/sj.ejhg.5201880; published online 20 June 2007

The 3rd International Meeting on Cryptic Chromosomal Rearrangements in Mental Retardation and Autism was held once again in Troina (Italy) on April 13 and 14, 2007. The scientific program was prepared by a Scientific Committee made up of Dr Bert de Vries (The Netherlands), Dr Samantha Knight (UK), Dr Christa Lese Martin (USA), and Dr Corrado Romano (also the local host in Troina).

After welcoming addresses from Professor Alessandra Renieri, on behalf of the European Society of Human Genetics, Professor Lidia Larizza, on behalf of the European Cytogeneticists Association, Dr Franca Dagna Bricarelli, on behalf of the Italian Society of Human Genetics, and Dr Maurizio Elia, Deputy Scientific Director of the IRCCS Associazione Oasi Maria Santissima, Dr Corrado Romano introduced the meeting on behalf of the Scientific Committee.

The program included three monothematic sessions (Mechanisms, Autism, and Phenotypes), one roundtable discussion, two short communication sessions, and a poster session.

In the Mechanisms Session, chaired by Mariano Rocchi (Italy), there were presentations from Nigel Carter (UK) on normal copy number variation $(\mathrm{CNV})$ and its impact on clinical array-based comparative genome hybridization $(\mathrm{aCGH})$, and from Heather Mefford (USA) on segmental duplications and genomic disorders: a targeted approach for disease discovery.

Dr Carter introduced the audience to the functional importance of CNVs within the human genome. He is a

*Correspondence: Dr C Romano, Unit of Paediatrics and Medical Genetics, Department for Mental Retardation, IRCCS Associazione Oasi Maria Santissima, Via Conte Ruggero 73, 94018 Troina (EN), Italy. Tel: + 390935 936285; Fax: + 390935 936533;

E-mail: cromano@oasi.en.it founding member of the International Structural Variation Consortium, which has screened all 270 individuals from the four HapMap populations with ancestry in Europe, Africa, and East Asia for CNV using two complementary technologies: the Affymetrix 500k SNP genotyping platform and aCGH to a whole genome tiling path array comprising $\sim 27000$ probes. This is the first global, genome-wide assessment of $\mathrm{CNV}$ and its relationship to haplotype structure and provides an important resource for understanding how genome structure impacts on biological function. The results of the effort can be summarized as follows: (1) the identification of over a thousand CNVs in these four populations, many of which are newly described, and which contain hundreds of genes and noncoding functional sequences; (2) the delineation of the linkage disequilibrium properties of hundreds of these CNVs, and the identification of tagging SNPs; and (3) the identification of genomic regions that show the most dramatic variation in copy number between populations and the exploration of selection as an explanation for these observations. To facilitate international collaboration in the analysis of patients with learning difficulties and/or dysmorphology using array technology, they have developed a WWW-based database (DECIPHER; http://decipher. sanger.ac.uk) that stores both patient phenotype and molecular data and allows visualization of genomic rearrangements within the genome browser Ensembl as well as documents 'normal' CNVs.

The focus of Dr Mefford's talk was genomic disorders caused by the presence of flanking segmental duplications that predispose these regions to recurrent rearrangement. On the basis of the duplication architecture of the genome, Dr Mefford and her colleagues from Professor Evan Eichler's Lab highlighted 130 genomic regions that they 
hypothesize are candidates for previously unreported genomic disorders. Using this approach, they have identified novel recurrent microdeletions and microduplications in a total of six regions. Using custom oligonucleotide arrays, they showed that the break points of these rearrangements lie in flanking segmental duplications that are frequently polymorphic. Interestingly, they have identified reciprocal events on both $16 \mathrm{p} 13$ and $17 \mathrm{q} 12$ in their two cohorts. In the case of 17q12, they identified the duplication not only in patients with mental retardation and epilepsy but also in normal relatives. The reciprocal deletion was seen in a case of prenatal multicystic renal dysplasia. They showed that deletion of this region also causes maturity onset diabetes of the young type 5 and pediatric renal disease. Characterization of additional patients with phenotypes similar to the probands has led to the identification of at least four novel genomic disorders in the human population related to childhood mental retardation, epilepsy, diabetes, and kidney disease.

In the Autism Session, chaired by Professor Lorenzo Pavone (Italy), Dr Mai Britt Giacobini (Sweden) presented the discovery of autism-risk loci through the identification of cryptic chromosomal rearrangements, and Professor Hans Hilger Ropers (Germany) presented CNV studies of autism and related disorders.

Dr Giacobini introduced the audience to the topic of autism spectrum disorders (ASD) and cryptic chromosome abnormalities. ASD are neurodevelopmental disorders characterized by impairments in communication and social interaction, accompanied by repetitive and stereotyped behaviors and interests. ASD are highly heritable and heterogenous disorders with a complex genetic etiology. The prevalence of ASD is $0.6 \%$, and in most cases the cause remains unknown. Despite extensive investigations that involved mutation screening along with linkage and association studies, mutations have only been detected in a few cases and no definite disease genes have yet been identified. Cryptic chromosome abnormalities, among them maternally derived duplications/triplications of chromosome 15q11-13, have been reported in patients with ASD. Testing using tiling resolution aCGH makes it possible to detect cryptic chromosome abnormalities that escape detection by conventional karyotyping. Using an array containing 33000 BAC clones, Dr Giacobini and coworkers have initiated tests on a cohort of patients with ASD - 24 patients with syndromic ASD and 9 patients with 'pure' ASD. To detect microdeletions or microduplications of autism candidate genes that escape detection by aCGH, they designed a multiplex ligation-dependent probe amplification (MLPA) synthetic probe set consisting of $\sim 30$ probes to test for gene-dose imbalances of candidate genes in patients with ASD. They found four potentially, clinically relevant imbalances: $1.4 \mathrm{Mb} \operatorname{dup}(7)(\mathrm{p} 22)$, $1.4 \mathrm{Mb} \operatorname{dup}(2)(\mathrm{q} 13), \quad 3.0 \mathrm{Mb} \operatorname{del}(17)(\mathrm{p} 13)$, and $1.6 \mathrm{Mb}$ del(22)(q11.2). To date, 90 ASD patients have been tested by MLPA, and there have been three findings: one confirmed and two probable duplications within chromosome 15q12. The conclusion of Dr Giacobini was that aCGH should be considered an essential aspect of the genetic analysis of patients with syndromic ASD.

Professor Ropers started his presentation from the consideration of autism as a complex disorder that need not necessarily be multifactorial. Genetic heterogeneity may play a major role, and the contribution of single gene defects has been underestimated. Submicroscopic deletions and duplications are not confined to Mendelian disorders but are also common risk factors for complex diseases. A high rate of de novo mutations and heterogeneity in complex diseases may explain the meager yield of previous association studies (the evolutionarily old major gene model is probably wrong). Clinically relevant deletions and duplications do not always cause disease (eg, TAR, $\operatorname{dup}(16)(\mathrm{p} 13)$ and $\operatorname{del}(22)(\mathrm{q} 11.2))$ and large imbalances may predispose to more than one condition. In addition, understanding the pathogenesis of microdeletion syndromes and recessive disorders represents a bottleneck in mutation screening since the identification of a genome imbalance using aCGH must lead to the mutation analysis of the second allele in recessive disorders.

The Phenotypes Session was divided into two parts: in the first part, chaired by Professor Dian Donnai (UK), Dr Bert de Vries (Netherlands) presented an overview of the novel microdeletion syndromes and Dr John Barber (UK) gave a presentation on the transmitted imbalances, autism, and arrays; in the second part, chaired by Dr Bert de Vries (Netherlands), the focus was on Williams syndrome or Williams-Beuren syndrome (WBS) with presentations from Professor Dian Donnai (UK) on phenotypic characteristics of $7 \mathrm{q} 11.23$ syndromes and clues to genetic localization, and from Dr May Tassabehji (UK) on the use of $3 \mathrm{D}$ imaging to provide clues regarding gene dosage in the WBS's critical region.

Dr de Vries opened his overview from the starting point that chromosome abnormalities are an important cause of mental retardation, detectable in $\sim 10 \%$ of cases, depending on the clinical selection criteria and techniques used. In recent years, new molecular cytogenetic techniques, such as fluorescence in situ hybridization (FISH), have led to the awareness that subtelomeric rearrangements below the level that can be detected by the light microscope $(<5 \mathrm{Mb})$ are also a significant cause of human malformations and mental retardation in 2-5\% cases. Recently new techniques, such as MAPH and MLPA, have become available to test the subtelomeric integrity. In contrast to FISH, both latter techniques also allow for detection of single subtelomeric duplications, thereby, increasing the yield of detection of subtelomeric abnormalities among the mentally retarded. Clinical preselection of patients can be achieved by using checklists (De Vries et al, 2001). Such a preselection is important when labor-intensive and 
time-consuming diagnostic techniques are used, but might be less relevant for more high-throughput screening methods. Targeting the telomeric regions using the various new techniques has led to the identification of novel microdeletion syndromes related to the telomeres, such as 1qter, 3qter, 9qter, and 22qter. In addition to microdeletions, novel microduplications of certain telomeric regions are also found. De Vries and colleagues found $5(0.6 \%)$ subtelomeric duplicatons among 834 mentally retarded individuals using MLPA: duplications of 5qter, 9pter, 9qter, 12 pter, and 18qter. So far, the latter are predominantly single cases, and, therefore, do not lend themselves to the recognition of overlapping clinical features that might lead to the characterization of a specific syndrome. For this reason, clinical collection of these rare cases with unbalanced chromosome aberrations is required and offered through the ECARUCA database (www.ecaruca.net). Using aCGH with a resolution of $1 \mathrm{Mb}(\sim 3500$ clones $)$, microdeletions and duplications elsewhere in the genome have been identified. More recently, tiling resolution arrays covering the whole genome have been used, leading to identification of microaberrations in $10-15 \%$ of mentally retarded patients with additional dysmorphisms. Novel syndromes are now being characterized, for example, the $17 q 21.3$ and 15q24 microdeletion syndromes. In both syndromes, specific genomic structures, namely identical segmental duplications, are the underlying cause of the recurrence of these genomic disorders. This novel, full coverage aCGH technique proves the importance of chromosomal microdeletions and duplications as a common cause of mental retardation and congenital abnormalities, and opens a new molecular karyotyping approach.

Dr Barber focused his talk on the role of transmitted imbalances that are compatible with a normal phenotype. Transmitted imbalances provide cytogenetic precedents, which indicate that deletions of gene poor and duplications of regions of moderate gene density are not always associated with disease. He is in charge of a Chromosome Anomaly Collection that can be accessed at www.ngrl.org. uk/Wessex/collection/, and contains 227 families to date. These include 149 cytogenetically visible, unbalanced chromosome abnormalities (UBCAs) and 78 euchromatic variants (EVs) that have been directly transmitted from parents to children. Among the 149 UBCAs, chromosome arms, 5p and 8p (with 20/149 (13\%) and 19/149 (13\%) anomalies, respectively), contain more transmitted anomalies than any other part of the genome. Among the 27 new families are:

1. Transmitted deletions of distal $3 p$ that are consistent with the idea of 'chromosomal non-penetrance'.

2. Transmitted deletions of medial $5 p$ that implicate the cadherin gene cluster in the developmental delay and behavioral problems found in six members of the same family.
3. Transmitted and de novo duplications of $8 \mathrm{p}$ that substantiate the existence of an 8p23.1 microduplication syndrome including Kabuki-like eyebrows as well as potential loci for autism.

4. Transmitted duplications of $6.2 \mathrm{Mb}$ within bands $12 \mathrm{q} 21.31$ to $12 \mathrm{q} 22$ including 48 genes with no phenotypic consequences.

Professor Donnai presented an update on $7 \mathrm{q} 11.23$ syndromes. She started by suggesting that research into the molecular pathology of WBS and efforts to link phenotypic features with specific genes in the deleted segment on $7 q 11.23$ have been slow due to the homogeneous nature of the deletion (that occurs by nonallelic homologous recombination between flanking LCRs). Her research group has found that the critical region for WBS features, other than heart defects associated with elastin haploinsufficiency, lies at the telomeric end of the deletion. It has been predicted that a reciprocal duplication of the region should occur at the same frequency as the deletion, especially since duplications have been defined in other microdeletion syndrome regions with similar genome architecture. Recently, there have been reports of patients with such duplications, and, interestingly, the patients have significant speech delay. This is in direct contrast to WBS patients who have fluent expressive language along with poor visuospatial skills. The paucity of reported cases of microduplications of 7q11.23 probably reflects ascertainment bias, milder effects, and limitations in diagnostic technology. The clinical presentation associated with reciprocal WBS duplications seems to be milder, and facial features are less distinct than those of WBS. It is intriguing that a distinctive part of both phenotypes concerns speech, which suggests that specific gene(s) in the region are exquisitely sensitive to dosage changes, and upsetting the balance can affect human speech and language as well as visuospatial capabilities.

Dr Tassabehji focused her presentation on the fact that many rare genetic syndromes underscore how facial abnormalities are a sign of defects in other parts of the body and how neurological abnormalities frequently accompany craniofacial dysmorphology. WBS is one such disorder where some 28 genes are homozygously deleted. The genome architecture at the WBS locus is rich in repetitive elements and segmental duplications, which makes the region susceptible to other chromosome rearrangements. Reciprocal WBS duplications do occur and have pathological consequences, albeit milder than deletions. Specific gene(s) in the region are therefore extremely sensitive to dosage changes and upsetting the balance affects craniofacial, visuospatial, speech, and language capabilities. Genotype-phenotype correlations have been difficult to assign, and position effects involving genes outside the critical region cannot be excluded. To further their studies, she and her co-workers have 
investigated rare individuals with atypical deletions in the WBS region, displaying partial or more severe clinical profiles, supplemented by the study of mouse models. The combination of clinical, psychological, and 3D-face morphometric analyses alongside detailed molecular profiling on a genome-wide scale has narrowed down the critical region harboring genes important for the WBS phenotypes as well as highlighting new regions associated with craniofacial and neurological development. Their goal is to gain a better understanding of the complex molecular pathways involved in human craniofacial and cognitive development using genetic disorders such as WBS to provide a way in.

The theme of the Roundtable Discussion, chaired by Professor Orsetta Zuffardi (Italy), was the use of genomewide arrays and new techniques in medicine. There were invited talks by Kim Smith (UK), Dr Joris Vermeesch (Belgium), and Dr Bert de Vries (Netherlands), and there was wide-ranging audience participation.

The Short Communications Session was also divided into two parts, the first with five presentations on mechanisms, chaired by Dr Frank Kooy (Belgium), and the second with six presentations on phenotypes, chaired by Dr Bert de Vries (Netherlands). The 3rd International Meeting on Cryptic Chromosomal Rearrangements in Mental Retardation and Autism consolidated and built upon to the success of the previous meetings. There were good discussions after each presentation, contributing to and promoting information sharing among the participants.

The 4th International Meeting on Cryptic Chromosomal Rearrangements in Mental Retardation and Autism is scheduled for April 4 and 5, 2008. 